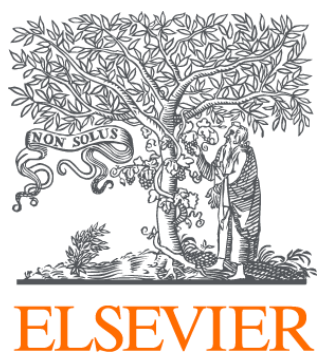

Since January 2020 Elsevier has created a COVID-19 resource centre with free information in English and Mandarin on the novel coronavirus COVID-

19. The COVID-19 resource centre is hosted on Elsevier Connect, the company's public news and information website.

Elsevier hereby grants permission to make all its COVID-19-related research that is available on the COVID-19 resource centre - including this research content - immediately available in PubMed Central and other publicly funded repositories, such as the WHO COVID database with rights for unrestricted research re-use and analyses in any form or by any means with acknowledgement of the original source. These permissions are granted for free by Elsevier for as long as the COVID-19 resource centre remains active. 


\section{Skin cancer plastic surgery during the COVID-19 pandemic}

\author{
Keywords: \\ COVID-19 \\ SARS-COV-2 \\ Skin cancer \\ Skin malignancies
}

\section{Dear editor}

Since the first cases occurring in Wuhan, China, in December 2019, the Coronavirus disease 2019 (COVID-19) quickly spread worldwide, being declared as pandemic by OMS. The number of infected people has rapidly increased, and on April 19th, 2020, it was estimated that it was present in 184 different countries accounting for more than 2,4 million confirmed cases. This scenario has created a dramatic burden over the healthcare systems that have been forced to set up new intensive care units and to create, within the existing hospitals, dedicated COVID-Hospitals, with separated clinical pathways for infected and non-infected patients. Such an emergency situation significantly reduced elective surgery, to limit as much as possible the risks of SARS-CoV-2 virus infection for both patients and medical and healthcare personnel and to leave the clinical facilities and intensive care available for a possible huge access of COVID patients.

Skin malignancies' priorities can be difficult to evaluate for plastic surgeon because skin cancer can range from very slowly progressing types that can be deferred, to aggressive forms.

Our Hospital is one of the most important cancer centres in Italy and, at the same time, one of the most important COVID-Hospital established for the pandemic. Then, a variety of clinical scenarios have been assessed by our group since the pandemic began. Although a careful selection of patients who are candidate for treatment by plastic surgeons is mandatory, there are no suggestions in the literature on which precise criteria the priority list should be based.

Until now, the risk of a significant worsening of the disease prognosis within 30 days has been the most important parameter for our priority list.

According to the cases treated using this principle, we believe that the following situations should be considered for surgery: 1) Patients with a recent excision of melanoma, who need to undergo re-excision and sentinel node biopsy (SNB). In case of positive SNB, these patients can access to specific adjuvant therapies, to be started as soon as possible to limit the risk of systemic relapse [1]; 2) Bleeding ulcerated skin cancer. Some skin malignancies may cause recurring episodes of bleeding and discharge requiring emergency room access, exposing the patient to complications and possibility to be infected with SARS-CoV-2 for the repeated entrance in the first aid; 3)Oncologic patients who are followedup for solid tumours (gynecological/breast cancer, melanoma, etc.) and develop solitary and resectable cutaneous/subcutaneous metastasis. In such cases, wide resection of metastases may improve patient overall survival; 4) Occurrence of rapidly growing and aggressive skin cancers (melanoma, angiosarcoma,etc.). Wide excision of these malignancies should be performed as early as possible; 5) Surgery should be also carefully evaluated for rapidly growing basal cell or squamous cell carcinoma. In rare cases, or in fragile patients such as transplant patients, the tumoral growth rate can be fast and, according to the anatomical sites, they can also invade surrounding and deep structures requiring major reconstructive procedures or causing functional permanent impairment [2]. Lid cancer can be included in this group and could be considered a medium priority surgery to be delayed only for a short period of time.

When managing patients undergoing surgery, some principles must be followed. Prior to admission to the Hospital/Department, screening for SARS-CoV-2 infection should be performed, by a nurse-directed triage, to exclude respiratory symptoms or recent contacts with affected people. The morning before surgery, each patient should undergo RT-PCR test on nasopharyngeal swabs. Then, the patient should be immediately hospitalized, to avoid the infection with SARS-CoV-2 after having undergone the test. During the same day the test results should be available and, if negative, the patient directed in a not-COVID clinical pathway and operating room(OR).In case of positive swab, surgical indication should be postponed [3], and with the use of telemedicine, the patient should be follow-up for worsening of the disease during home quarantine and re-evaluated with swab after confirmed infection clearance.

In case of an emergency situation such as bleeding the surgical procedures cannot be deferred and a dedicated in-patient unit, clinical pathway and OR should be available for SARS-CoV-2 surgical patients. Specific counselling about the increased risks of undergoing surgery during the COVID-19 incubation period should be done [4].

\section{Declaration of competing interest}

None.

\section{References}

[1] Santonocito C, Concolino P, Lavieri MM, Ameglio F, Gentileschi S, Capizzi R, et al Comparison between three molecular methods for detection of blood 
melanoma tyrosinase mRNA. Correlation with melanoma stages and S100B LDH, NSE biochemical markers. Clin Chim Acta 2005 Dec;362(1-2):85-93.

[2] Ferro M, Deodato F, Macchia G, Gentileschi S, Cilla S, Torre G, et al. Short-course radiotherapy in elderly patients with early stage non-melanoma skin cancer: a phase II study. Canc Invest 2015 Mar;33(2):34-8.

[3] Lei S, Jiang F, Su W, Chen C, Chen J, Mei W, et al. Xia Z Clinical characteristics and outcomes of patients undergoing surgeries during the incubation period of COVID-19 infection. Clin Med 2020 Apr 5:100331.

[4] Bracaglia R, D'Ettorre M, Gentileschi S, Tambasco D. Was the surgeon a satisfactory informant? How to minimize room for claims. Aesthetic Surg J 2014 May $1 ; 34(4): 632-5$

Stefano Gentileschi*

Fondazione Policlinico Universitario A. Gemelli IRCCS, Dipartimento Scienze Della Salute Della Donna E Del Bambino E Di Sanità Pubblica, Unità di Chirurgia Plastica, Roma, Italy

Università Cattolica del Sacro Cuore, Facoltà di Medicina e Chirurgia, Dipartimento di Medicina e Chirurgia Traslazionale, Roma, Italy

Anna Amelia Caretto Università Cattolica del Sacro Cuore, Facoltà di Medicina e Chirurgia, Dipartimento di Medicina e Chirurgia Traslazionale, Roma, Italy

Luca Tagliaferri

Fondazione Policlinico Universitario A. Gemelli IRCCS, Dipartimento di Diagnostica per immagini, Radioterapia Oncologica ed Ematologia -
Gemelli ART (Advanced Radiation Therapy), Interventional Oncology Centre (IOC), Rome, Italy

Marzia Salgarello

Fondazione Policlinico Universitario A. Gemelli IRCCS, Dipartimento Scienze Della Salute Della Donna E Del Bambino E Di Sanità Pubblica, Unità di Chirurgia Plastica, Roma, Italy

Università Cattolica del Sacro Cuore, Facoltà di Medicina e Chirurgia, Dipartimento di Medicina e Chirurgia Traslazionale, Roma, Italy

Ketty Peris

Fondazione Policlinico Universitario A. Gemelli IRCCS, Dipartimento Scienze Mediche e Chirurgiche, UOC Dermatologia, Roma, Italy Università Cattolica del Sacro Cuore, Dermatologia, Rome, Italy

* Corresponding author. Largo Francesco Vito 1, 00168, Roma, Italy. E-mail addresses: stefanogentileschi@gmail.com, stefano.gentileschi@policlinicogemelli.it (S. Gentileschi).

27 April 2020

Available online 1 May 2020 